\title{
Predicting Malignancy in Entirely Solid-appearing Adnexal Masses on Gray-Scale Ultrasound Based on Additional Ultrasound Findings, Clinical Complaints and Biochemical Parameters: A Retrospective Study
}

\author{
Jesús Utrilla-Layna, Begoña Olartecoechea, María Aubá, Alvaro Ruiz-Zambrana, Laura Pineda, Juan Luis Alcázar
}

\begin{abstract}
Purpose: To determine which clinical, biochemical and other sonographic parameters could be useful to predict malignancy in sonographically solid adnexal masses.

Materials and methods: Clinical, biochemical and other sonographic features from 227 women diagnosed as having an entirely solid adnexal mass on B-mode gray-scale ultrasound were reviewed for this retrospective study. All patients had undergone surgery and mass removal. Definitive histologic diagnosis was available in all cases. All parameters were compared to final histological diagnosis (benign or malignant) in univariate statistical analysis. Then a stepwise forward logistic regression analysis was performed to identify those features that independently predict malignancy.
\end{abstract}

Results: A total of 227 masses were analyzed. Patients' mean age was 52.9 years (range: $15-84$ years). A total of 150 masses were malignant and 77 were benign. In 52 cases carcinomatosis was observed on ultrasound scan and all theses cases were malignant. In the remaining 175 cases, univariate analysis showed statistical differences between benign and malignant tumors all parameters except tumor's volume. After logistic regression analys is tumor contour, acoustic shadowing, amount of color and CA 125 were identified as independent predictors of malignancy. Using a logistic model $94.4 \%$ of malignant tumors and $85.7 \%$ of benign tumors would be correctly identified.

Conclusion: The presence of carcinomatosis is invariably associated to malignancy. In absence of carcinomatosis, irregular tumor contour, absence of acoustic shadowing, moderate or abundant blood flow and a high CA 125 are associated to malignancy.

Keywords: Solid adnexal mass, Malignancy, Ultrasound.

How to cite this article: U trilla-Layna J, Olartecoechea B, Aubá M, Ruiz-Zambrana A, Pineda L, Alcázar J L. Predicting Malignancy in Entirely Solid-appearing Adnexal Masses on Gray-Scale Ultrasound Based on Additional Ultrasound Findings, Clinical Complaints and Biochemical Parameters: A Retrospective Study. Donald School J Ultrasound Obstet Gynecol 2013;7(1):80-85.

\section{Source of support: Nil}

Conflict of interest: None declared

\section{INTRODUCTION}

Entirely solid adnexal masses constitute a diagnostic challenge for most sonologists and sonographers. ${ }^{1} \mathrm{~A}$ significant number of these adnexal masses are malignant ${ }^{2,3}$ but most of them are benign. ${ }^{4,5}$ Therefore, accurate differential diagnosis is essential for adequate referral and optimal management. ${ }^{6-8}$

Most studies involving the use of ultrasound for discriminating benign from malignant solid adnexal masses focus on ultrasound findings of the mass itself, whereas other factors such as clinical complaints, physical examination or other additional ultrasound findings have not been analyzed in detail.

Our hypothesis is that these factors may predict malignancy in entirely solid adnexal masses. Thus, in the present study we aimed to determine whether any clinical, biochemical or other sonographic parameter could be hel pful for predicting malignancy in solid adnexal masses.

\section{MATERIALS AND METHODS}

This is a retrospective study that comprises a consecutive series of women diagnosed as having an entirely solid adnexal mass on gray-scale ultrasound evaluated and treated at our institution between J anuary 1995 and J une 2011. IR B approval was obtained for this study. However, patients' informed consent was waived due to the retrospective design as well as for all tests performed was routine for diagnostic workup for adnexal masses.

Patients' records including clinical, biochemical and sonographic data were reviewed. For ultrasound data, ultrasound reports as well as digital stored images were reviewed.

In our institution diagnostic workup for all patients diagnosed as having an adnexal mass include complete medical history, pelvic examination, and serum CA 125 levels determination, except for simple cysts, as well as an ultrasound evaluation.

For this study, medical history focused on patient's complaints, age and menopausal status. Clinical data recorded were as follows:

1. Patient's complaints: A symptomatic. Symptoms nonsuggestive of ovarian cancer such as pelvic painfor example, suggestive of endometriosis or abnormal uterine bleeding. Symptoms suggestive of ovarian cancer, such as abdominal swelling, bloating and abdominal discomfort. 
2. Menopausal status: Premenopausal or postmenopausal. Menopause was defined as 1 year of absence of menstruation in women older than 45 years. If hysterectomy had been performed, a woman was considered as postmenopausal if older than 50 years.

Pelvic exam was performed by staff specialists in Obstetrics and Gynecology. Findings were stated as follows:

1. 'Inconclusive', when no reliable information could be obtained.

2. 'Nonsuspicious', in the presence of a less than $8 \mathrm{~cm}$ maximum diameter adnexal mass, mobile at examination, of cystic or solid consistency but regular contours and no evidence of ascites.

3. 'Suspicious', in the presence of at least one of the following: Fixed and/or irregular adnexal mass regardless the size, evidence of ascites (fluid wave).

Transvaginal ultrasound evaluation was performed according to a predetermined protocol. ${ }^{9}$ In cases of large tumors, presence of fluid in Douglas' pouch or suspicion of pelvic implants transabdominal ultrasound was also performed. A lthough IOTA consensus defined a solid tumor as a tumor being $80 \%$ or more solid, ${ }^{10}$ in our study only tumors entirely solid ( $100 \%$ solid appearance) at B-mode examination (Fig. 1) were included in this study. Ultrasound parameters assessed were as follows:

1. Tumor volume was estimated according to the prolate ellipsoid formula $(A \times B \times C \times 0.5233)$ and expressed in $\mathrm{ml}$.

2. Tumor contour, stated as ' regular' (Fig. 1) or 'irregular' (Fig. 2).

3. A coustic shadowing; stated as 'absent' or 'present' (Fig. 3).

4. Bilaterality, stated as 'yes' or ' no'. In those women with bilateral masses we chose the largest one for statistical analysis.

5. The presence of ascites ('yes' or 'no'); defined subjectively by the examiner as the presence of fluid in pelvis surrounding the uterus and/or upper abdomen (Fig. 4).

6. The presence of tumoral implants-carcinomatosis-in pelvis or upper abdomen, stated as 'present' or 'absent' (Figs 5 and 6).

7. Subjective amount of flow within the mass based on color or power Doppler assessment; stated as 'absent', 'scanty' (Fig. 7), 'moderate' or 'abundant' (Fig. 8).

In all cases blood samples had been collected to measure CA 125 plasma concentration. CA 125 measurements were performed using an enzyme immunoassay with a monoclonal antibody (Cobas-Core CA $125 \mathrm{II}$, Laboratories Roche, B asel, Switzerland).

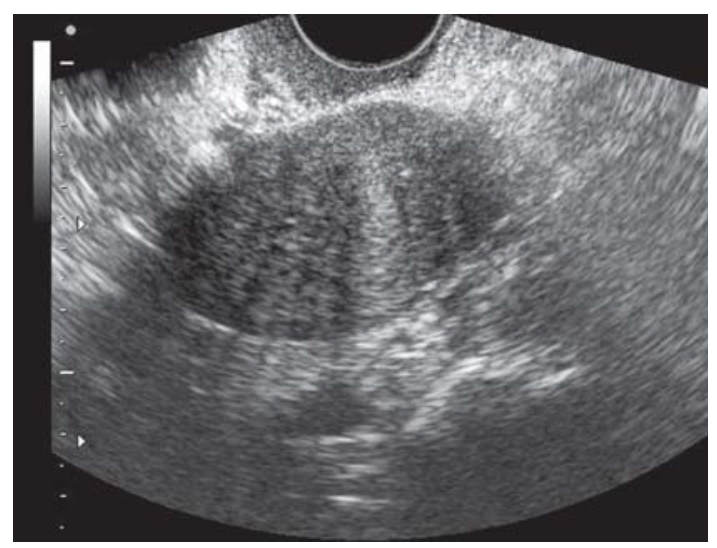

Fig. 1: Small solid adnexal mass with regular contour in a postmenopausal woman. Histopathology revealed an ovarian fibroma

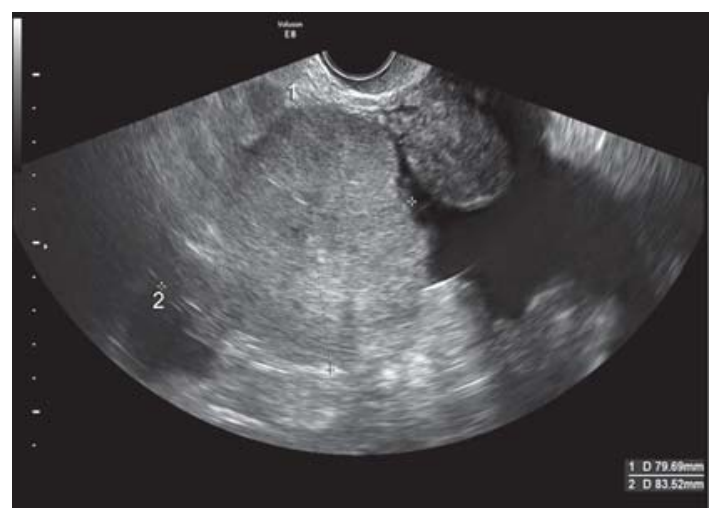

Fig. 2: Large irregular solid mass in a postmenopausal woman. Ascites is also seen. Histology revealed a primary serous ovarian carcinoma

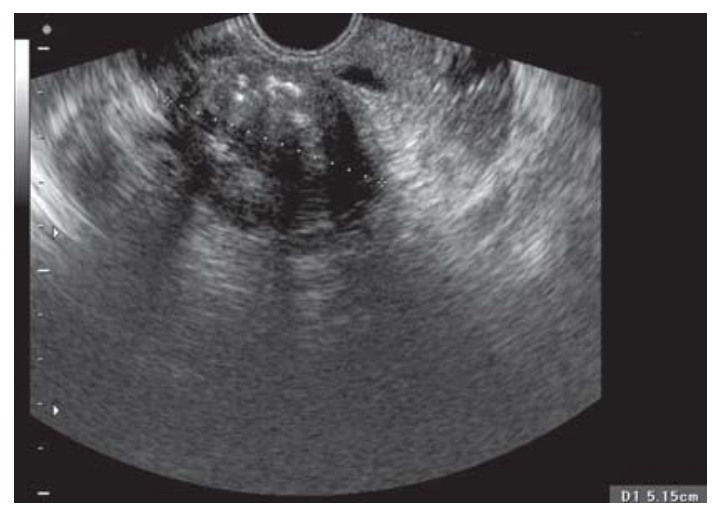

Fig. 3: Transvaginal ultrasound depicting a well-defined solid mass with acoustic shadowing. Histology revealed a benign ovarian Brenner tumor

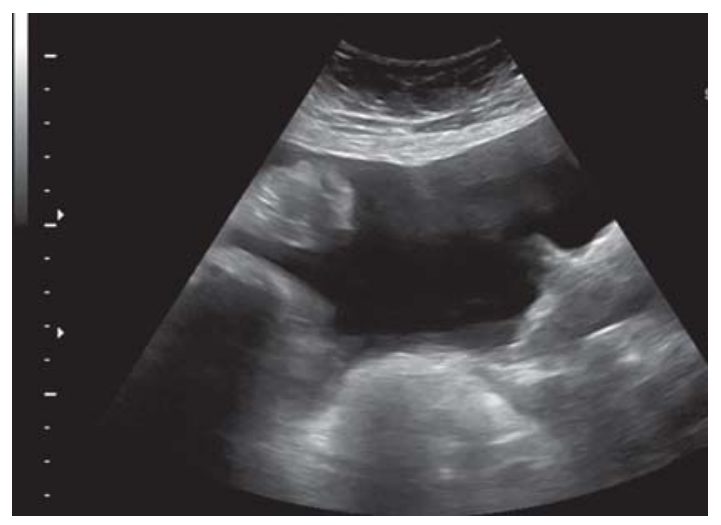

Fig. 4: Transabdominal ultrasound depicting a significant amount of ascites within the abdomen and pelvis 


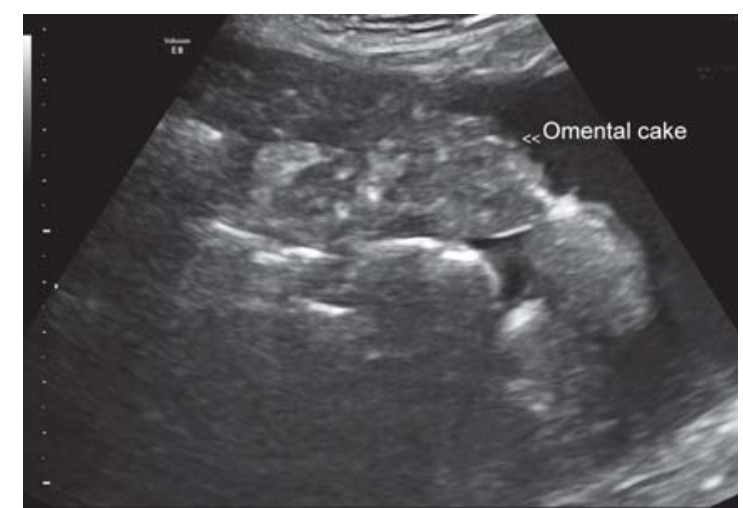

Fig. 5: Transabdominal ultrasound depicting tumoral involvement of the greater omentum (omental cake)

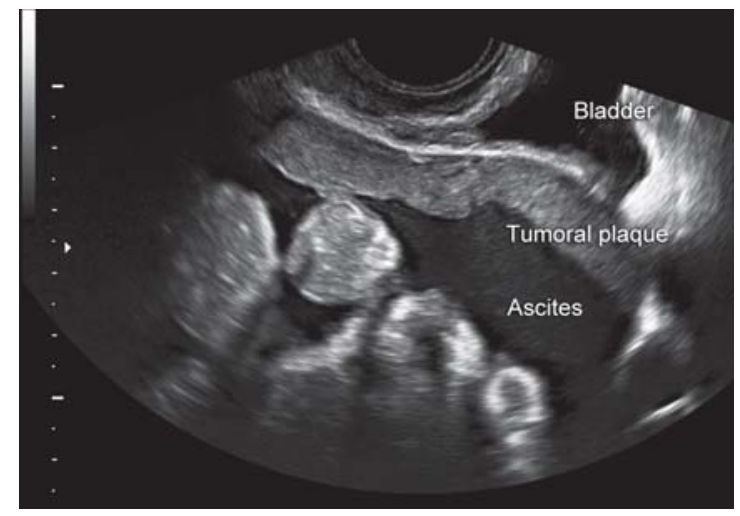

Fig. 6: Transvaginal ultrasound that shows pelvic carcinomatosis, a tumoral plaque is seen involving the bladder peritoneum

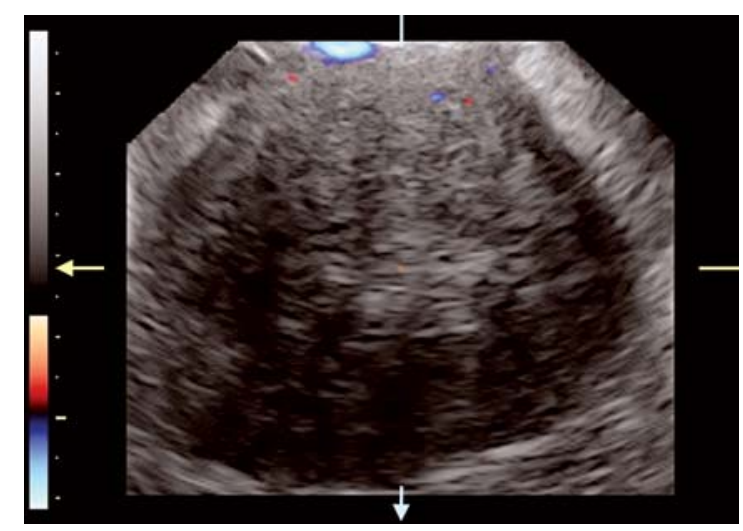

Fig. 7: Transvaginal color Doppler ultrasound from a solid mass with scanty vascularization. Histology revealed a benign ovarian fibrothecoma

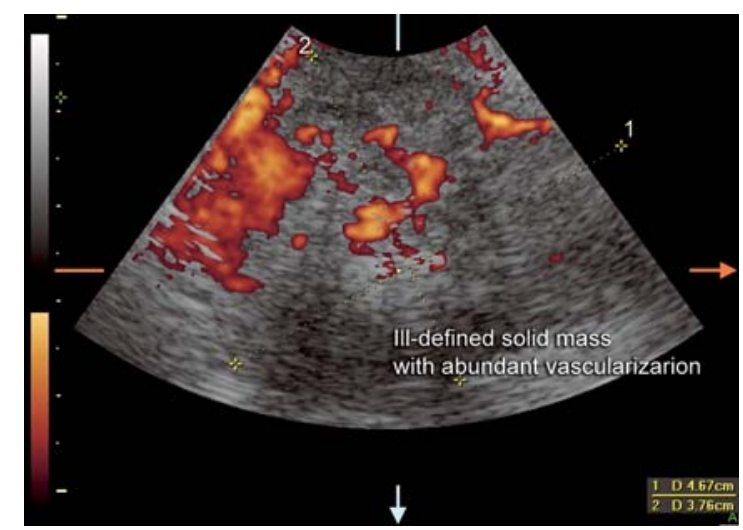

Fig. 8: Transvaginal power Doppler ultrasound from an ill-defined large solid pelvic mass with abundant vascularization. Histology revealed a malignant ovarian Sertoli-Leydig cell tumor
All tumors were surgically removed and definitive histological diagnosis obtained. Tumors were classified according to the World Health Organization. ${ }^{11}$ Ovarian malignancies were staged according to the FIGO. ${ }^{12} \mathrm{~L} \mathrm{ow}$ malignant potential tumors were considered as malignant.

The only inclusion criterion was the diagnosis of an entirely solid mass. Exclusion criteria were as follows: No complete medical, laboratory or ultrasound records; surgery not performed in our institution.

\section{STATISTICAL ANALYSIS}

We firstly performed univariate statistical analysis to find statistical differences in the parameters assessed between malignant and benign tumors. The Kolmogorov-Smirnov test was used to assess normal distribution of continuous data. Continuous data were compared using the one-way ANOVA or Mann-Whitney $U$ test, according to their distribution. Categorical variables were compared using the Chi-square test for dichotomous variables and the K endall's tau-b test for ordinal variables.

Once univariate analysis was done, we performed multivariate forward stepwise logistic regression analysis (MLR) including as independent variables only those variables found to be statistically different between malignant and benign tumors in the univariate analysis and as dependent variable the final diagnosis (malignant or benign). For M LR analysis dichotomous variables were stated as follows: M enopause ( $1=$ yes, $0=$ no), physical exam ( 1 = suspicious, $0=$ nonsuspicious or inconclusive), symptoms ( 1 = suspicious, $0=$ nonsuspicious $)$, ascites ( $1=$ yes, $0=$ no), bilaterality $(1=$ yes, $0=$ no), tumor contour ( $1=$ irregular, $0=$ regular $)$, acoustic shadowing $(1=$ no, $0=$ yes), amount of flow ( $1=$ moderate/abundant, $0=$ absent/ scanty) and the CA 125 and tumor volume value were log transformed. The Hosmer-L emeshow test was used to assess the goodness-to-fit of the model. ${ }^{13}$

A $p$ value $\leq 0.05$ was considered as statistically significant. All statistical analyses were performed using the SPSS 15.0 statistical package (SPSS Inc, Chicago, II).

STROBE guidelines were used for reporting this study. ${ }^{14}$

\section{RESULTS}

Three-hundred and twelve patients were eligible for this study. We excluded 85 women for the following reasons: 52 women underwent surgery out of our hospital and 33 women had incomplete records. Thus, 227 women were ultimately included in the study.

Patients' mean age was 52.9 years old (SD: 13.8), ranging from 15 to 84 years old. 
Ninety-one (40\%) women were premenopausal and 136 $(60 \%)$ were postmenopausal.

One hundred and fifty $(66 \%)$ tumors were proved to be malignant and 77 (34\%) were benign (Table 1).

A fter univariate analysis all parameters were statistically different between benign and malignant lesions (Table 2).

We observed that in all 52 cases with evidence of carcinomatosis in ultrasound examination tumors were malignant and none were benign ( $100 \%$ specificity). So,

Table 1: Histologic diagnoses of those masses included

\begin{tabular}{lrr}
\hline & N & $\%$ \\
\hline Endometrioma & 2 & 0.9 \\
Teratoma & 24 & 10.5 \\
Cystadenofibroma & 2 & 0.9 \\
Tubo-ovarian abscess & 2 & 1.3 \\
Fibroma/fibrothecoma & 24 & 10.5 \\
Uterine leiomyoma & 14 & 6.2 \\
Brenner tumor & 6 & 2.6 \\
LMP carcinoma & 2 & 0.9 \\
Ovarian cancer* & 104 & 46.3 \\
Metastatic cáncer** & 47 & 20.7 \\
\hline Total & 227 & 100.0 \\
\hline
\end{tabular}

*E pithelial ovarian cancer-94; Sex-cord stromal tumors-2; Germ cell tumor-4; Primary lymphoma-2; S arcoma-2; **Breast- 11 ; Colon sigmoid-11; Stomach-10; Lung-2; Uterine leiomyosarcoma-2; Endometrium-1; Liver-1; Appendix-1; Biliary tract -1 ; Ureter -1 we decided to focus the analysis in those cases without sonographic evidence of carcinomatosis ( $n=175$ ).

Out of those 52 women with carcinomatosis, 44 (84\%) had primary ovarian cancers (two stage II, 35 stage III and 7 stage IV) and eight (16\%) had metastatic tumors to the ovary.

Univariate analysis in the remaining 175 cases showed statistical significant differences for all variables except for tumor volume (Table 3).

However, after MLR analysis only amount of blood flow, tumor contour, acoustic shadowing and log CA 125 were identified as independent predictors for malignancy (Table 4).

Using the model developed $[Z=-6.236+27.334 \times$ (amount of color) $+6.006 \times$ (acoustic shadowing) +3.193 $\times$ (tumor contour) $+5.842 \times(\log C A 125)] 94.0 \%$ of malignant tumors and $85.7 \%$ of benign tumors were correctly identified (Table 5).

\section{DISCUSSION}

In this study, we aimed to assess whether any clinical, biochemical or other ultrasound parameters could be helpful for predicting malignancy in solid-appearing adnexal masses on gray-scale ultrasound. We found that carcinomatosis is

Table 2: Univariate statistical analysis for all 227 masses

\begin{tabular}{llrr}
\hline Parameter & Benign $(\mathrm{n}=77)$ & Malignant $(\mathrm{n}=150)$ & $\mathrm{p}$-value \\
\hline Patient's age (years)* & $48.6(14.4)$ & $55.1(13.0)$ & $<0.001$ \\
Symptoms of OC & $9 / 77(11.7 \%)$ & $68 / 150(45.3 \%)$ & $<0.001$ \\
Postmenopausal status & $36 / 77(46.8 \%)$ & $100 / 150(66.7 \%)$ & 0.003 \\
Suspicious physical examination & $16 / 77(20.8 \%)$ & $101 / 150(67.3 \%)$ & $<0.001$ \\
Median CA 125 (IU/mI)** & $18.3(5.0-1760.0)$ & $231.7(5.6-31494.0)$ & 0.005 \\
Median tumor volume (ml)** & $54.0(1.8-3453.8)$ & $75.6(1.5-1776.0)$ & 0.118 \\
Ascites & $9 / 77(11.7 \%)$ & $93 / 150(62.0 \%)$ & $<0.001$ \\
Bilaterality & $5 / 77(6.5 \%)$ & $43 / 150(28.7 \%)$ & $<0.001$ \\
Acoustic shadowing & $35 / 77(45.5 \%)$ & $10 / 150(6.7 \%)$ & 0.001 \\
Irregular contour & $16 / 77(20.8) \%$ & $107 / 150(71.3 \%)$ & $<0.001$ \\
Moderate or abundant flow & $12 / 77(15.6 \%)$ & $126 / 159(84.0 \%)$ & $<0.001$ \\
Signs of carcinomatosis & $0 / 77(0 \%)$ & $52 / 150(34.7 \%)$ & $<0.001$ \\
\hline
\end{tabular}

OC: Ovarian cancer; ${ }^{*}$ Expressed as mean, standard deviation (SD) in parentheses; **Expressed as median, range in parentheses

Table 3: Univariate statistical analysis for 175 masses without carcinomatosis

\begin{tabular}{llll}
\hline Parameter & Benign $(\mathrm{n}=77)$ & Malignant $(\mathrm{n}=98)$ & $\mathrm{p}$-value \\
\hline Patient's age (years)* & $48.6(14.4)$ & $53.8(13.4)$ & 0.014 \\
Symptoms OC & $9 / 77(11.7 \%)$ & $41 / 98(41.8 \%)$ & 0.001 \\
Postmenopausal status & $36 / 77(46.8 \%)$ & $60 / 98(61.2 \%)$ & 0.039 \\
Suspicious physical examination & $16 / 77(20.8 \%)$ & $59 / 98(60.2 \%)$ & 0.001 \\
Median CA 125 (IU/ml)** & $15.4(5.0-1760.0)$ & $128.0(5.6-1,4247.0)$ & 0.001 \\
Median tumor volume (ml)** & $54.0(1.8-3453.8)$ & $75.3(1.5-1,776.0)$ & 0.095 \\
Ascites & $9 / 77(11.7 \%)$ & $47 / 98(48.0 \%)$ & 0.001 \\
Bilaterality & $5 / 77(6.5 \%)$ & $20 / 98(20.4 \%)$ & 0.007 \\
Irregular contour & $16 / 77(20.8) \%$ & $62 / 98(63.3 \%)$ & 0.001 \\
Moderate or abundant flow & $12 / 77(15.6 \%)$ & $82 / 98(83.7 \%)$ & 0.001 \\
Acoustic shadowing & $35 / 77(45.5 \%)$ & $6 / 98(6.1 \%)$ & 0.001 \\
\hline
\end{tabular}

OC: Ovarian cancer; ${ }^{*}$ Expressed as mean, standard deviation (SD) in parentheses; **Expressed as median, range in parentheses 
J esús Utrilla-Layna et al

\begin{tabular}{llccc}
\hline \multicolumn{5}{c}{ Table 4: Multivariate logistic regression analysis } \\
\hline Variable & Coefficient & Odds ratio & $95 \% \mathrm{Cl}$ & $\mathrm{p}$-value \\
\hline Moderate/abundant flow & 3.308 & 27.334 & $8.429-88.646$ & 0.001 \\
Absence acoustic shadow & 1.793 & 6.006 & $1.389-25.808$ & 0.016 \\
Log CA 125 & 1.765 & 5.842 & $2.312-14.765$ & 0.001 \\
Irregular tumor contour & 1.161 & 3.193 & $1.002-10.381$ & 0.049 \\
\hline
\end{tabular}

Table 5: Agreement between histology and predictive model in solid adnexal masses without ultrasound signs of carcinomatosis

\begin{tabular}{llcc}
\hline & \multicolumn{2}{c}{ Histology } \\
\cline { 3 - 4 } & & Benign & Malignant \\
\hline Predictive model & Benign & 66 & 6 \\
& Malignant & 11 & 92 \\
\hline
\end{tabular}

best predictor for malignancy. In cases with no carcinomatosis detected on ultrasound, irregular tumor contour, absence of acoustic shadowing and elevated CA 125 are predictor of malignancy.

Our study has some limitations. First, when facing with a solid adnexal mass the differential diagnosis should be established between those lesions from ovarian origin, benign or malignant, and other lesions from extraovarian origin, such as benign uterine leiomyoma or cancers from other origin. ${ }^{15}$ In our study, according to ultrasound reports and stored digital images all tumors were considered to be as adnexal origin. However, we had 14 cases of pedunculated uterine myomas.

Pedunculated uterine leiomyomas can be easily recognized when both ovaries are identified independently from the tumor and the tumor is seen arising from the uterine wall. ${ }^{16,17}$ This may be considered as a limitation in our study since many asymptomatic pedunculated leiomyomas seen in our institution during study period did not undergo surgery, thus our results may overestimate malignancy rate of entirely solid pelvic masses.

A nother limitation of our study is that we did not assess interobserver agreement for some subjective ultrasound features such as tumor contour or the assessment of amount of blood flow.

Finally, we included cases of truly cystic lesions (endometriomas, teratomas, tubo-ovarian abscess). Certainly, these masses may exhibit 'solid' appearance but indeed they are not solid. We recognize that including these cases could bias our results.

Studies focusing on solid adnexal or pelvic masses are scanty. Some authors have reported that clinical assessment may be useful for the differential diagnosis. ${ }^{15}$ However, many women present asymptomatic and the tumors are accidentally found out during routine examination. As a matter of fact, in our study patients' complaints and pelvic exam findings were not independent predictors for malignancy.
Regarding the clinical implications of our findings we found that the presence of carcinomatosis on B-mode ultrasound was invariably associated with malignancy and no single benign tumor had this finding. This finding could be expected but our study confirms that in presence of carcinomatosis other features of the tumor are less relevant and the patientshould be referred to a gynecologic oncologist. Furthermore, this finding should prompt a thorough transabdominal ultrasound in the presence of a solid tumor in transvaginal ultrasound. Some studies have shown that carcinomatosis can be assessed by ultrasound. ${ }^{18,19}$

In cases with no evidence of carcinomatosis, some sonographic tumor features are helpful to discriminate benign from malignant solid adnexal tumors. The presence of moderate or abundant blood flow within the tumor and/ or irregular tumor contour as well as the absence of acoustic shadows are predictive for malignancy. A s a matter of fact, acoustic shadowing has been reported as a common finding in some types of benign solid ovarian tumors. ${ }^{20,21} W$ hereas irregular solid tumor has been associated to malignancy. ${ }^{22}$

A nother interesting issue is the use of CA 125. Recent studies have shown that CA 125 does not add relevant information to ultrasound for discriminating betw een benign and malignant adnexal masses. ${ }^{23,24}$ However, according to our data CA 125 could be helpful if a solid mass with no evidence of ascites or carcinomatosis is found out at ultrasound examination.

Regarding the generalizability of our findings, it is difficult to determine since this is a retrospective study and our data need to be confirmed in prospective studies.

\section{CONCLUSION}

We have shown that malignancy can be predicted in entirely solid adnexal tumors analyzing other ultrasound features such as the presence of carcinomatosis, tumor contour, acoustic shadowing and amount of flow.

\section{REFERENCES}

1. Funt SA, Hann LE. Detection and characterization of adnexal masses. Radiol Clin N orth A m 2002;40:591.

2. Guerriero S, A lcazar J L, Coccia M E, et al. Complex pelvic mass as a target of evaluation of vessel distribution by color D oppler sonography for the diagnosis of adnexal malignancies: Results of a multicenter European study. J Ultrasound Med 2002;21:1105.

3. B rown DL, D oubilet PM, M iller FH, et al. B enign and malignant ovarian masses: Selection of the most discriminating gray-scale and Doppler sonographic features. Radiology 1998;208:103. 
4. Jeong $Y Y$, O utwater EK, Kang HK. Imaging evaluation of ovarian masses. Radiographics 2000;20:1445.

5. Valentin L, Hagen B, Tingulstad S, Eik-Nes S. Comparison of 'pattern recognition' and logistic regression models for discrimination between benign and malignant pelvic masses: A prospective cross validation. Ultrasound Obstet Gynecol 2001;18:357.

6. A merican College of Obstetricians and Gynecologists. A COG Practice Bulletin. M anagement of adnexal masses. Obstet Gynecol 2007;110:201.

7. Guerriero S, A jossa S, G arau N, et al. U Itrasonography and color Doppler-based triage for adnexal masses to provide the most appropriate surgical approach. Am J Obstet Gynecol 2005; 192:401.

8. Alcázar JL, Royo P, Jurado $M$, et al. Triage for surgical management of ovarian tumors in asymptomatic women: A ssessment of an ultrasound-based scoring system. U Itrasound Obstet Gynecol 2008;32:220.

9. Alcázar JL, Ruiz-Perez M L, Errasti T. Transvaginal color Doppler sonography in adnexal masses: Which parameter performs best? U Itrasound O bstet Gynecol 1996;8:114.

10. Timmerman $D, V$ alentin $L, B$ ourne $T H$, et al. International Ovarian Tumor A nalysis (IOTA) Group. Terms, definitions and measurements to describe the sonographic features of adnexal tumors: A consensus opinion from the International Ovarian Tumor A nalysis (IOTA) Group. Ultrasound Obstet Gynecol 2000;16:500.

11. Serov SF, Scully RE, Sobin LH. International histological classification of tumors ( $\mathrm{V}$ ol 9). Histological typing of ovarian tumors, W orld Health Organization, Geneva, 1973.

12. Sheperd JH. Revised FIGO staging for gynecological cancer. BrJ Obstet Gynecol 1989;96:889.

13. Hosmer DW, Lemeshow S. A pplied Logistic R egression. N ew Y ork: Willey-Interscience, 1989.

14. von EIm E, A Itman DG, Egger $M$, et al. The Strengthening the Reporting of O bservational Studies in E pidemiology (STROBE) statement: Guidelines for reporting observational studies. L ancet 2007;370:1453.

15. Brown DL. A practical approach to the ultrasound characterization of adnexal masses. U Itrasound Q 2007;23:87.

16. Sladkevicius $P, V$ alentin $L, M$ arsál $K$. Transvaginal Doppler examination for the differential diagnosis of solid pelvic tumors. J Ultrasound M ed 1995;14:377.

17. Brown DL, Dudiak KM, Laing FC. Adnexal masses: US characterization and reporting. Radiology 2010;254:342.

18. Savelli L, De Iaco P, Ceccaroni M, et al. Transvaginal sonographic features of peritoneal carcinomatosis. Ultrasound Obstet Gynecol 2005;26:552.

19. Testa AC, Ludovisi M, Savelli $L$, et al. Ultrasound and color power Doppler in the detection of metastatic omentum: A prospective study. Ultrasound Obstet G ynecol 2006;27:65.
20. Paladini D, Testa A, V an Holsbeke C, et al. Imaging in gynecological disease (5): Clinical and ultrasound characteristics in fibroma and fibrothecoma of the ovary. U Itrasound Obstet Gynecol 2009;34:188.

21. Dierickx I, Valentin L, V an Holsbeke $C$, et al. Imaging in gynecological disease (7): Clinical and ultrasound features of Brenner tumors of the ovary. Ultrasound Obstet Gynecol 2012; $40: 703$.

22. Timmerman D, Testa AC, B ourne T, et al. Simple ultrasoundbased rules for the diagnosis of ovarian cancer. Ultrasound Obstet Gynecol 2008;31:681.

23. V alentin $L$, Jurkovic $D, V$ an $C$ alster $B$, et al. Adding a single CA 125 measurement to ultrasound imaging performed by an experienced examiner does not improve preoperative discrimination between benign and malignant adnexal masses. Ultrasound Obstet Gynecol 2009;34:345.

24. A lcázar J L, Guerriero S. Gray-scale ultrasound versus CA 125 levels for predicting malignancy in adnexal masses. Int J Gynaecol Obstet 2011;114:290.

\section{ABOUT THE AUTHORS}

Jesús Utrilla-Layna

Department of Obstetrics and Gynecology, Clinica Universidad de Navarra, U niversity of Navarra, Pamplona, Spain

\section{Begoña Olartecoechea}

Department of Obstetrics and Gynecology, Clinica Universidad de Navarra, U niversity of Navarra, Pamplona, Spain

\section{María Aubá}

Department of Obstetrics and Gynecology, Clinica Universidad de Navarra, U niversity of Navarra, Pamplona, Spain

\section{Alvaro Ruiz-Zambrana}

Department of Obstetrics and Gynecology, Clinica Universidad de Navarra, U niversity of N avarra, Pamplona, Spain

\section{Laura Pineda}

Department of Obstetrics and Gynecology, Clinica Universidad de Navarra, U niversity of N avarra, Pamplona, Spain

\section{Juan Luis Alcázar (Corresponding Author)}

D epartment of O bstetrics and Gynecology, ClinicaU niversidad deN avarra, A venida Pio XII, 36, 31008 Pamplona, Spain, Phone: 34-948-255400 Fax: 34-948-296500, e-mail: jlalcazar@unav.es 